\title{
A Simplified Approach to Determining Resolution of Optical, Ion and Electron Microscope Images
}

\author{
A. E. Curtin, R. Skinner, and A.W. Sanders \\ National Institute of Standards and Technology, Boulder, CO 80305
}

We have developed a simplified approach for calculating the resolution of images taken across a wide variety of magnifications and a wide variety of tools. The simplest definition of resolution is a measure of the minimum resolvable distance between two points, commonly expressed by the Rayleigh criterion for diffraction-limited systems. In practice, however, when microscope vendors and users check the resolution of an instrument, a standard sample is used and the measurement is made from the image. A variety of additional methods exist, including calculation of the contrast transfer function, Fourier analysis of image, and calculations of optimal probe size.[1] We would like to provide a routine for determining image resolution, using the sigmoidal function, or logistic equation. For image analysis, we are concerned with the resolution of an image independent of its collection method. Similarly, a method that is simple, easily interpretable and well-defined is very important when directly comparing images. We present a simple analytical fit of bright-dark transitions in microscope images using the logistic equation:

$$
S(x)=c+\frac{a}{1+\operatorname{Exp}\left[\frac{-x+x_{0}}{b}\right]}
$$

The relevant fit parameter is given here as $b$, which is proportional to the image resolution as modified by a prefactor. We relate $b$ to a practically defined resolution using high angle annular dark-field scanning transmission electron microscopy (STEM) images of the Si [110] dumbbell from an aberration corrected STEM. The STEM image allows for quantitative comparison of known dimensions to fix the prefactor on $b$, giving the proportionality of the image resolution to the fit parameter.

The intent of the STEM fit is to ground our sigmoidal curve (and therefore the prefactor) in a standard sample with known spacing. Although our model is designed to work on edge features across many methods of collection, the determination of the prefactor rests in the Gaussian fit of the standard Si[110] dumbbell. In Figure 1 , we fit the $\mathrm{Si}[110]$ dumbbell spacing to the sum of two Gaussian functions of the same width, $\sigma$, but with different locations.[3] The dumbbell spacing is known to be $0.136 \mathrm{~nm}$, making the image resolution roughly 60 $\mathrm{pm}$ to $70 \mathrm{pm}$, or $\sigma$ scaled by a factor of 1.5.[2] The Si[110] sample is ideal in this case as the dark gap in the middle of the dumbbell at $67 \mathrm{pm}$ is reliably resolved by our microscope, giving us a real-world judge of resolution with which to determine the prefactor on $b$. The parameter $b$ in the logistic equation was analytically related to $\sigma$ from the Gaussian fit. Combining this relation with the known spacing in $\mathrm{Si}[110]$, we are able to find the prefactor, calculating image resolution $=3.53 \mathrm{~b}$.

By grounding our analysis in the quantitative analysis of a STEM HAADF image of Si[110], we are able to directly calculate resolution from the sigmoidal fits of contrast edges for a wide variety of microscope images. Figure 2 shows that, once we have fixed our prefactor on $b$, the calculated resolutions provided by our sigmoidal fit closely follow the Rayleigh criterion for a series of optical images collected over a range of numerical apertures (NA). We can harness this method to track changes in resolution with changes in defocus and other microscope parameters, and to back out effective probe size in scanned beam systems. We will show how this analysis may be applied to microscopes here at NIST Boulder Laboratories, allowing for the quantification of resolution directly from images, covering magnification ranges from optical to STEM methods. Additionally, we 
will discuss the mathematical equivalency of our fit to other analytical methods for calculating resolution, including Gaussian fits, linear fits, and error functions. [4-5].

\section{References:}

[1] D. C. Joy, J. Michael, and B. Griffin, Proc. of SPIE (2010) p 76383J.

[2] Y. Kohno, et al. Microsc. Microanal. 17, (2011) p 1162.

[3] Image courtesy of Toshihiro Aoki, Arizona State University, unpublished.

[4] B. Rieger and G. N. A. van Veen, EMC 2008 14th European Microscopy Congress 1-5 September 2008, Aachen, Germany, ed. M. Luysberg, K. Tillmann, and T. Weirich (Springer Berlin Heidelberg, Berlin) p 613.

[5] This work is a contribution of NIST, an agency of the U.S. government and as such is not subject to copyright in the United States.

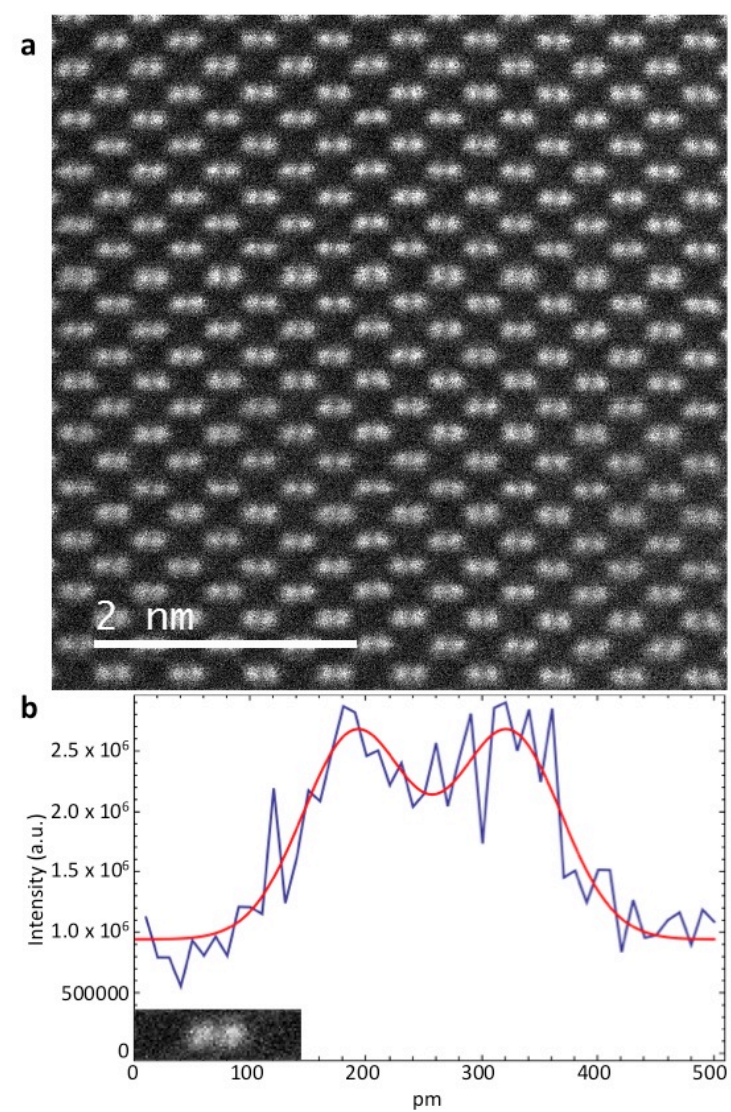

Fig 1: STEM image of Si[110] dumbbells. Any random dumbbell may be fit to the sum of two Gaussians, each of the same width.

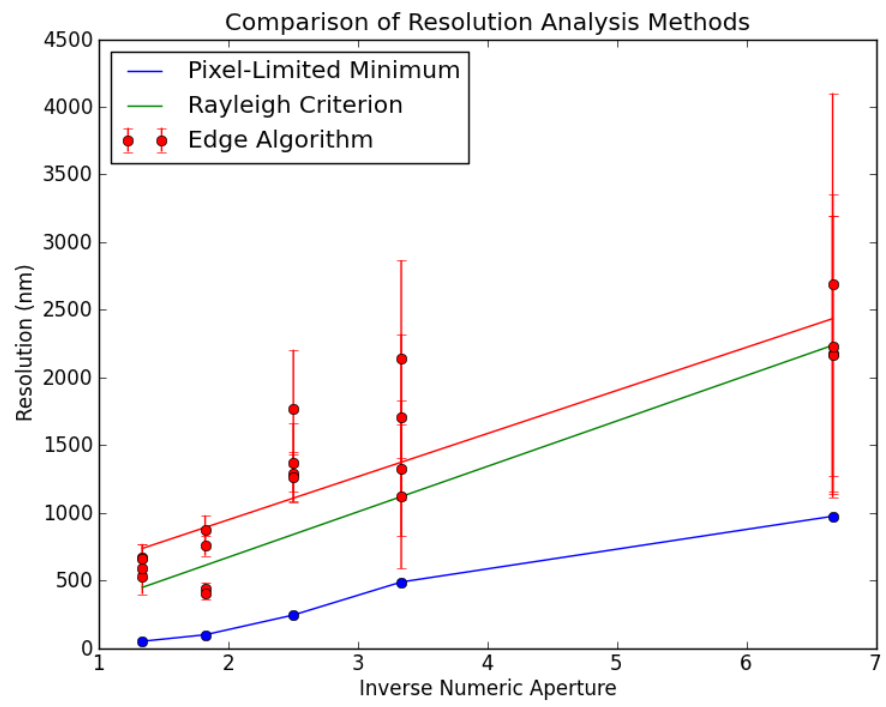

Fig 2: The edge algorithm for output for multiple optical images is plotted vs. 1/NA. The model (red line) closely follows the Rayleigh criterion (green). The pixel-limited minimum resolution is shown in blue. 